\title{
UCritical 那igest.
}

\section{SEMEIOLOGY OF SLEEP.}

\author{
BY DR. PAUL BLOCQ.
}

Chef des travaux Anatomo-Pathologiques à la Salpétrière.

Translated from the original MS. by H. W. Marett Tims, M.D. (Edin.)

IN a large number of maladies, natural sleep presents. various modifications, such as insomnia, twitchings and startings, morbid dreams and nightmares, the semeiological value of which we shall first consider.

Again, there exist states of true pathological sleep; nypnotism, vertigo with paralysis, narcolepsy, hysterical sleep, hypnotic sleep, somnambulism-which are more or less analogous to sleep, and of which we shall in the second part point out the differential characteristics.

\section{I.}

Insomnia is the more or less complete privation of sleep. As a general rule it depends upon conditions which keep up cerebral excitability. In this respect it is necessary to remember that the duration of natural sleep is variable, according to individual idiosyncracies.

Although insomnia appears in the majority of acute and chronic diseases, it is, in certain cases, none the less a symptom of real importance.

Certain causes, predisposing to insomnia, are well recognised; emotional persons and old men are particularly subject to it; so also, occupations which necessitate a large amount of brain work or compel late hours, would be equally predisposing causes.

"This paper forms part of a treatise on the "Symptomatology of Nervous Diseases," which will shortly be published (in conjunction with $T$. Onanoff), by BI. Masson in Paris. 
The determining causes have been divided according as they consist of excitations arising in the nerve centres themselves, or as the result of peripheral impressions transmitted by the nerves; or lastly, as they affect the brain by means of an alteration in the blood.

The majority of affections of the brain itself or of its membranes are causes of insomnia. Nevertheless, some of them only appear to act by the painful sensations to which they give rise ; in others, loss of sleep appears as an essential symptom.

Among the former it will be sufficient to mention meningitis, encephalitis, cerebral syphilis and tumours. In the two last cases the insomnia acquires a certain semeiological value, as it is evidence of the intensity of the headache, of its nocturnal oharacter, and may, perhaps, be a guide as to its duration.

Among the latter group one may mention the com mencement of a great number of cases of mental alienation, and it is from this point of view, namely of diagnosis, that insomnia is interesting to consider here.

Among the peripheral excitations, pain comes in the first rank, whether caused by a wound or neuralgia. A certain number of cutaneous irritations may also be mentioned. All these instances, which it would be wearisome to enumerate, are only inıportant with regard to their differentiation.

Alterations of the blood, which give rise to insomnia, may in the first instance consist only of disorders of the circulation, and from this point insomnia may also be of great diagnostic value, for it is important to know that it is frequently the first sign of an attack of syncope. Alterations of the blood due to infections and intoxications cause sleeplessness. Among the former we may mention, in particular, typhoid fever, of which sleeplessness is often an initial symptom ; also acute secondary jaundice is frequently ushered in by insomnia. Among intoxicants it is scarcely necessary to mention coffee and tea.

Lastly, there exists a kind of essential insomnia generally, it is true, appearing in those persons with a hereditary nervous temperament in whom it is impossible VoL. XrV. 
to point to any direct cause to which one may, with any precision, attribute the trouble,

Twitchings, Startings.-In natural and sound sleep the body is in a state of relaxation, inert, and subject only to the laws of gravity. Nevertheless, by reason of the special mode of cerebral action, which characterises it, the excito-motor power of the cord is increased, which gives rise to exaggeration of reflex activity and to the frequency of twitchings and jerkings, the semeiological value of which is thereby greatly diminished.

In this respect they may be considered as transitional phenomena with regard to insomnia, of which they represent the slighter forms, proceeding from the same causes.

Dreams.-Between certain dreams or visions, which may be considered normal, and nightmare, the pathological nature of which is sufficiently indicated by the name " nocturnal asthma," which is quite as frequently applied to it, the limit is so slightly defined-at least from the point of view of their symptomalogical value-that we can study both under the name of " morbid dreams."

This point of pathology has not yet been examined as it ought to be. However, it should be noticed that the majority of the maladies have a slow onset, and their symptoms remain for a long time unnoticed. Now it is possible that though unnoticed in the waking state, the pathological disturbance becomes sensible during sleep, and thus produces dreams to which their relations with the affected organ give a real semeiological value. On the other hand, dreams assume very special characteristics in certain neuroses (hysteria), intoxications (alcoholism); in fact, in those already predisposed they may have an influence upon the form of the nervous affections (hysterical paralysis, hallucinations, forms of mental aberration).

In the disturbances of the circulatory apparatus the dreams are frequently very short, and terminated abruptly by waking with a start, They bear upon tragic events, are accompanied by anxiety, and fears of death are prominent.

Affections of the respiratory organs produce horrible 
dreams. They are those in which one notices nightmare in the pathological sense of the term. The dream is often about animals, which the dreamer imagines he sees seated on his chest, or fastened to his throat, followed by extremely painful sensations of suffocation and anguish. Certain kinds of dreams also are more or less usual in persons the subjects of disturbances of the digestive organs, more especially of the stomach. According to several authors, the nocturnal terrors of children point to intestinal indigestion. Dreams associated with disorders of digestion are usually connected with gustatory hallucinations accompanied by sensations of weight in the epigastrium.

But it is most especially in nervous affections that dreaning acquires a semeiological importance. The repetition for some time prolonged, of the same nightmare, is by itself equivalent to what is called "signs of degeneration." There are cases of this kind in which a nightmare instals itself, so to speak, permanently, and for years regularly torments the person. On the other hand, in certain morbid conditions such as hysteria, one notices a constant habit of terrifying dreams with visions of animals, just like those of alcoholism. In these cases the dreams may be followed by paralysis or abasia. It is also to be noticed that in cases of paraplegia of a similar nature it is unusual for the patients to dream of walking.

Finally, it is necessary to mention the dreams in hysterotraumatism, which are almost always said to give rise to the neurosis and have such constancy that they should be enumerated amongst the symptoms. Let it be added, that mental aberration and general paralysis may be ushered in by strange dreams curious in relation with the ulterior hallucinations, and that the dream, in this respect, would have a certain influence over the development of the delirium.

As to alcoholic intoxication, it is known that it produces among other symptoms frightful dreams of animals, rats, mice, \&c., at other times the dreams relate to the occupation of the patient. However that may be, the peculiarities of the dream have sufficient importance in the diagnosis of alcoholism to prevent negligence in seeking for them. 
II.

We must classify as pathological sleep a certain number of states more or less analogous to sleep but very different. from natural sleep, and it is very important to know how to differentiate the one from the other. They are, hypnotism, the vertigo of paralysis, narcolepsy, hysterical sleep, hypnotic. sleep and somnambulism.

The sleeping sickness (MacCarthy), or "sleeping dropsy" of negroes, is prevalent on the western coast of Africa.

Three degrees can be distinguished between the tendency to drowsiness and complete lethargy. The first symptom of the disease consists in the eyelids being half closed at the same time the person is taken with a fit of drowsiness, at different times of the day; then these fits become almost continuous, the patient with difficulty aroused to eat, and falls asleep again almost immediately. Finally, the sleep becomes persistent, and different excitations no longer have power to arouse the patient, who thus succumbs after a sleep lasting for three or four days, presenting in certain cases a notable diminution of temperature. This affection is unaccompanied by motor or sensory disturbances, and has a mean duration of three or four months. The.characters of this condition are so pronounced, and its geographical distribution even so exact, that it is unnecessary to discuss its differential diagnosis.

Paralysing vertigo has been observed endemically in Switzerland, and appears to be a modified form of the preceding affection.

This affection is characterised by headache, vertigo, enfeeblement of the muscular system, with staggering gait and double ptosis. The disease lasts some months, and always terminates favourably. In relation to this, it is convenient to mention another variety, described by Garget, occurring in a person who, after an explosion, was seized with violent excitement accompanied by diplopia and visual troubles. Following on this, double occulo-motor paralysis with ptosis, progressive muscular weakness with intellectual apathy and tendency to sleep. There were no paralytic or 
sensory troubles. The patient after some relapses and remissions slept for five months and then died. This affection seems to be related to acute poliencephalitis (Mauthner) ; it is comparatively rare, but it is, nevertheless, necessary to be warned of its existence in order to avoid confusion with the following forms.

Narcolepsy would not constitute a pathological entity but only a symptom (unlike insomnia), possibly appearing in widely divergent cases. It consists essentially in a pathological exaggeration of the desire to sleep. The tendency to sleep is constantly seen in the youth of both sexes; it is. irresistible, comes on for the most part suddenly, it recurs sometimes at very short intervals, and with sufficient strength to cause the patient to yield inevitably as soon as it is felt (Ballet). The tendency to sleep is sometimes spontaneous, at others it is brought about by the most diverse external influences, movements or emotions. It is more or less intense as the case may be, varying from an apathetic condition to deep sleep. This sleep has in other respects a normal appearance. The patient does not dream, and be can only be awakened by the most energetic excitations.

The condition of narcolepsy has been met with in a.certain number of cases in which it was impossible to make out any other morbid alteration; most frequently it is a question of cardiac affection, of dilatation of the stomach, of general obesity, of diabetes, or of hereditary neuropathy.

The diagnosis of narcolepsy would in itself be easy, but it is sometimes more difficult to make out the morbid condition giving rise to it. For this purpose one should not neglect to make a careful examination of the heart, digestive, functions, and more especially of the urine, for this has proved the presence of diabetes which up to that time had been unrecognised. We consider that hysterical sleep, as well as the different varieties of hysterical somnambulism which some authors have classed as narcolepsy, has sufficiently pronounced symptoms of its own to merit a special description. Indeed, we place hysterical apoplexy along with hysterical sleep, as it. is impossible to differentiate them nosographically, according to the teaching of $\mathrm{M}$. Charcot, for 
it is a question of one and the same morbid condition; the only difference being that in a case described under the name of hysterical apoplexy (Debove and Achard), the sleep attack has a sudden onset and is followed by hemiplegia, while this paralysis may be absent in other cases.

In a great number of observations the attack of sleep is preceded or followed by usual phenomena or by premonitory indications of a convulsive attack and habitual hysteria. At other times the onset is sudden, and analogous to that of apoplexy due to cerebral hæmorrhage, and one may see patients who are attacked by it fall suddenly, it may be in the midst of a conversation, or in the midst of their occupation.

Again, the sleep crisis may come on after the attack. The sleep having once come on, the patient usually has a placid expression, but sometimes the features are a little drawn ; the respiration is not stertorous, and the patient does not snore. The limbs may be quite limp, or, perhaps, more frequently in a state of general rigidity; it may attack the lower extremities only, or even a single limb.

One constantly notices a kind of vibration of the eyelids, the more so on attempting to open the eyes, which converge upwards and inwards. Trismus exists in almost all cases (Gilles de la Tourette). Respiration is regular, the pulse quiet, the temperature may rise a degree and some tenths (38.9), but never more. The urine is passed in the bed, and it has a chemical composition characteristic of the convulsive attack; it is diminished in quantity and in its constituents, and there is an inversion of the formula for the phosphates (Gilles de la Tourette and Cathelineau). ${ }^{1}$ The sleep is also characterised by this fact, namely, the strongest excitations-noises, inhalations of irritating vapours, faradisation of the skin, nerve trunks and of the musclesproduce no reaction. It is equally noticeable that during

1 These authors have observed that the relative proportion of the earthy phosphates to the alkaline phosphates contained in nomal urine is 1 to 3 ; but in hysterical urine during the attack the proportion is equal. It is this alteration that they refer to by the term "inversion of the formula of the phosphates." 
the sleep one often sees the patient execute certain.movements, as opisthotonos, movements of salutation, \&c., at regular intervals without awaking.

Lastly, there sometimes exist hyperæsthetic points which are at the same time hysteria-producing zones, pressure upon which brings on an attack which puts an end to sleep.

This condition should be differentiated from natural sleep, from coma, and from the other pathological conditions of sleep previously mentioned.

Natural sleep terminates under the influence of various excitations of the senses-a fact which is sufficient to distinguish it. The knowledge of the antecedents and of the commencement of the attack, if it was ushered in by an hysterical crisis, would usually be sufficient to establish the diagnosis; but in the absence of these points hesitation would be possible. One would then have to rely on the general aspect of the patient, on the temperature, on the presence of rigidity (trismus, contracture, palpitation of the eyelids) and on the alterations of the sensibility, in order to diagnose it from coma.

In narcolepsy the sleep is neither accompanied nor followed by hysterical phenomena; it presents none of the preceding characteristics. In the following paragraph we shall see in what way it is distinguished from hypnotic sleep.

Hypnotic sleep presents very various phenomena, according as it is observed in different inäividuals, and, moreover, it assumes very distinct forms. It can be divided at the outset into grand hypnotism, petit hypnotism.

The "grand hypnotisme" (Charcot) occurs in hysterical persons. It comprises three periods or phases, each possessing somatic characters peculiar to itself; $(a)$ the lethargic state ; $(b)$ the cataleptic state; (c) the somnambulic state.

We shall only describe here the two first states, the third being more appropriately considered in the paragraph below, under the heading of somnambulism. The commencement of the lethargic period of the grand hypnotism is frequently marked by a deep inspiration, accompanied by a slight laryngeal bruit, and it is not uncommon, at the same time, 
for a slight froth to appear on the lips. The eyes are closed, the eyelids vibrate slightly; the head, the trunk, and the whole muscular system are in a state of complete relaxation; anæsthesia is complete. The characteristic sign of this state is the property which the muscles possess of contracting under the influence of mechanical stimuli, applied either to the muscles themselves or to the nerves distributed to them (the nearo-muscular hyper-excitability of Charcot). If a certain amount of pressure be applied to the belly of a muscle, it soon contracts, and this contraction only disappears on working up the antagonistic muscles. Again, pressure applied to the trunk of a nerve produces immediate contraction of all the muscles supplied by that nerve, and this contraction yields, like the preceding one, under the same influence.

These symptoms are more or less marked according to the individual, and they are sufficient to affirm the diagnosis. However, this same tendency to contraction may exist in hysterical sleep, but then, if one were so enlightened as to the facts, one would diagnose the lethargic state by proving that a slight breath of air on the face quickly dissipates it. The cataleptic stage of the grand hypnotism is characterised by the complete immobility of the subject, and, at the same time, by the peculiarity that the limbs remain in any position in which they may be placed. The eyes are open, the eyelids do not blink, tears run down the cheeks, the gaze is fixed, and the face is immovable. Anæsthesia is complete, as in the preceding state. If an arm be raised, or if the body be placed in any attitude, whatever the position thus assumed is maintained for a considerable time without effort. Moreover, there exists a kind of suggestion by the muscular sense-that is to say, that the attitudes in which the limbs are placed give rise to corresponding expressions on the face. If, for example, the hands of the subject are placed in an attitude of prayer, the countenance becomes ecstatic.

Hypnotic catalepsy is comparatively easy to diagnose from these signs. Simulation can be detected by proving the fact that in true catelepsy the limbs retain the position in which they are placed without any sign of effort on the 
part of the patient, as acceleration of the respiratory movements. In hysterical sleep one sometimes observes the same peculiarity of the limbs to retain their position, but then these positions do not cause corresponding expressions of the countenance as in hypnotic catalepsy. In cases of catatonia sufficient indications are given by the facts, and the mental condition of the subject.

The "petit hypnotisme" has various degrees-drowsiness, light sleep, deep sleep, very deep sleep, somnambulism (Bernheim). In the first degree it is only a question of heaviness and numbness; in light sleep, the persons sleep but hear any convarsation that is taking place; in deep sleep they lose all remembrance of what has happened during sleep, but they are still in relation with those who are present as well as with the operator. In very deep sleep the isolation of the person is complete, and he is in relation with the operator only.

In addition to these signs there are also present anæsthesia and more especially a marked docility to the suggestions of the operator. This last characteristic would be sufficient to establish the diagnosis of hypnotism; but in all cases of the petit hypnotism, in the absence of objective symptoms, one must rely on the good faith of the patient.

Somnambulism comprises a certain number of conditions, all characterised by an automatic activity of the nerve centres, which causes those who are the subjests of it to perform unconsciously a variety of different actions.

We shall adopt for the description the following classification (Charcot's) of somnambulisms :

Physiological ........................................... Natural s.

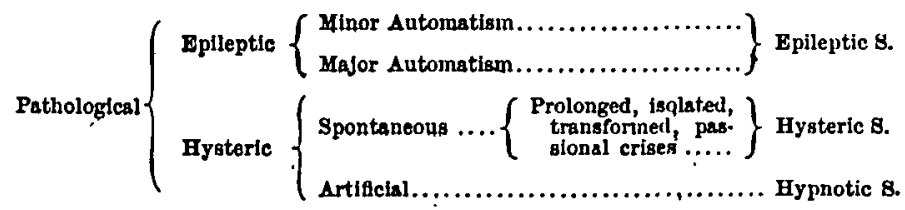

Natural or physiological somnambulism has long been recognised. The somnambulist of this variety, according to the etymology of the word, is "one who walks during sleep." 
It has been defined thus: "Somnambulism is when the functions performed during the period of wakefulness are repeated during sleep, which, in other respects, is natural "

It is seen in children and usually occurs in the middle of the night. The person goes to bed as usual and after some hours' sleep, suddenly, or after a slight momentary restlessness, gets up; then during a longer or shorter period he performs very various actions, goes back again to bed, and on awaking has no recollection of what has taken place during the night.

The somnambulist has his eyes wide open, but keeps them fixed, the pupils are contracted and he guides himself without hesitation, apparently only seeing the objects or persons which are playing a part in the episode he is enacting. Some somnambulists appear to hear; this fact, however, is less well-established; when spoken to in a loud voice they generally take no heed, and continue the actions they have begun.

The muscular sense is remarkably developed in them, and it is to the hyperæsthetic condition of this sense that the facility with which they often extricate themselves from perilous positions, as jumping, balancing themselves on the roof, \&o,, is to be attributed. Moreover, the tendency to spasimodic muscular contractions, which mark the hysterical somnambulist, does not occur in these cases.

On awaking-and this is a very important point-the somnambulist has completely forgotten all that he has done during the attack. He performs very different actions, but speaking in a general way, he always appears to be acting a dream, which compríses one or more of a series of quite distinct episodes.

From these signs it is comparatively easy to distinguish natural somnambulism, if one takes into account the entire normal condition of the patient in the interval between, and the nocturnal character of, the attacks. These characters differ from those of epileptic somnambulism. Every form of epilepsy comprises a species of automatism, usually rudimentary, which may, under certain conditions, acquire considerable development. 
The simple and most common forms are those which partake of the nature of the petit mal, the petit automatisme. Sometimes the trouble in these cases lasts only a few moments, and then the patient continues, for instance, the work with which he was occupied at the onset of the attack. At other times the fit is of longer duration and more complex. In a case given by Trousseau, a magistrate sitting at a learned society suddenly goes out of the hall without his hat, walks a hundred yards outside, then returning takes part in the discussion without having any recollection of what he had done.

Cases of this description are comparatively easy to diagnose, for the psychical and impulsive characters of these attacks are seldom the only ones which mark the subject of them. Most frequently he has true vertigo, or even convulsive epileptic seizures, and, in a certain number of these cases, these signs immediately precede the psychical disturbance.

The difficulty is much increased when other forms are in question, epileptic ambulatory automatism (Charcot). The attack rnay last from two hours to six days, during which the patient performs a series of capricious movements with a desire to walk about; he is quite unconscious, and has the appearance of an ordinary man, but retains no recollection of his movements. The diagnosis would be easy should the patient have any history of antecedent convulsive crises, and above all if the onset of the period of unconsciousness is marked by an aura.

But it may not be so very easy. The diagnosis is then based rather on the negative symptoms-absence of hysterical signs and crises-and on the analogies presented by these unconscious impulses, and those which follow the fit of sleep; and lastly, it is by noting most carefully the positive results of treatment with bromide, that we should be justified in designating these cases as epileptic somnambulism. Hysterical somnambulism is marked by attacks which are usually preceded or followed by the motor phenomena of a hysterical fit, but more rarely these are manifested some time previously. 
Hysterical somnambulisms may be considered as transformations of the phase of the writhing attitudes of the hystero-epileptic attack. This phase is characterised by delirium, in which the patient, by his words and gestures, appears to have hallucinations of various descriptionscheerful, sad, mournful, terrifying, \&c. The animated performances in which these persons are then taking part usually commence in the same order and proceed in a kind of series.

In the first group of acts there is an hysterical attack in which the convulsive period is shortened and the emotional phase is lengthened and simulates somnambulism. When the attack is coming on it begins with some epileptiform movements of short duration; then the phase of delirium supervenes, bringing with it, as usual, a succession of scenes always the same. It is then that the patient is a true somnambulist; his eyes are open, at least most usually, but he only sees the dream which he is following outhe walks, gesticulates, talks, cries or sings. Sometimes a word or certain noises may occur during the delirium, but they only serve to modify the order of succession of the phases; no new one is added, that is to say, no suggestion has any creative influence. Finally, it is often possible to bring on an attack by hypnotism, or, on the other hand, it can be arrested by pressure upon the hystero-inhibiting zones.

In another variety of seizure there is no abnormally prolonged phase of the attack, but a completely isolated phase. The patient enters upon the scene which he is about to enact by some twisting movements of the hands, which represent the shortened convulsive period of the attack; he then proceeds to the performance of certain actions in accordance with the hallucinations from which he is suffering.

In this case external intervention has now more effect not only in modifying the scenes of the delirium, without, however, adding anything new to it, but it elicits answers from the patient to questions put to him. Otherwise the patient is not amenable to suggestion, and shows none of the 
contracture of somnambulism; but his attitude is more quiet, his activity diminished, his passivity increased.

And finally, in the last class are the cases usually concidered as examples of " double identity." These are cases of people living, so to speak, a two-fold existence; that is to say, passing alternately from one state to another, so that everything that happens in state No. 1 is only known in a similar state, and the same thing occurs in state No. 2.

The subject in reality exists as two personalities, each unknown to the other and differing in psychical and sometimes even in physical characteristics. In fact, there may exist in one of these states hysterical symptoms (hemianæsthesia, contracture, \&c.), which may disappear in the other condition.

It is evident that all cases of this kind have characters so prominent, that there is no need to dwell more upon the diagnosis of them.

Hypnotic somnambulism is the third stage of the grand hypnotism (Charcot), or the fifth degree of the petit hypnotism (Bernheim).

This state is marked by less spontaneous activity on the part of the subject, and his passive obedience to every suggestion which it may please the experimenter to make to him. There is also present a marked exaltation of the muscular power, and, above all, of the special senses.

The general sensibility is usually diminished, or entirely absent. The eyєs are wide open, and the subject appears to be awake, but in the grand hypnotism there is a physical sign which allows us to ascertain the reality of this state. This sign consists in a muscular hyper-excitability, which may be produced by a superficial excitation of the skin. A breath, or the slightest tickling, causes contracture, which disappears by manipulation exercised on the antagonising muscles. On awaking, the somnambulist has forgotten all that has passed, but he may retain the recollection during another period of somnambulism. Lastly, there remain a large number of conditions called traumatic amnesia, which have certain points of resemblance with the automatic states which we have just described, but their nosographical 
history is not yet sufficiently made out to warrant their description here. Nevertheless, their eventuality is foreseen, and ideas as to their causation would then establish the diagnosis. 